\title{
Minimally Invasive Endoscopic Spine Surgical Techniques for Treating Radiculopathy Secondary to Sacroiliac JoInt Fusion Complications: Case Series
}

\author{
Ralf Wagner, MD ${ }^{1}$ \\ Albert E. Telfeian, MD, PhD²
}
Background: Minimally invasive SI joint fusion has increased in popularity. A possible complication that can result from the procedure is a radiculopathy (sacral or lumbar) that is caused by placement of the $\mathrm{SI}$ joint fusion implant.
Case Report: Two cases are presented: A 60-year-old woman with a S1 radiculopathy secondary to a cortical bone fragment contacting the $\mathrm{S} 1$ nerve, and a 62-year-old man with a $\mathrm{L} 5$ radiculopathy and the anteriorly directed SI joint fusion implant positioned anteriorly and compressing on the $L 5$ nerve. Both patients had resolution of their symptoms with endoscopic treatment.

Conclusion: An endoscopic treatment is suggested here for radiculopathy secondary to minimally invasive SI joint fusion.

Key words: Endoscopic spine surgery, lumbar radiculopathy, minimally invasive spine, SI joint fusion

\section{BACKGROUND}

The treatment of sacroiliac (SI) joint pain is multimodal and involves treatment specialists from internal medicine, physiatry, interventional pain management, and spine surgery. Anti-inflammatory medication, physical therapy, steroid injections, and radiofrequency ablation are all effective treatments. When SI joint pain is not successfully treated with nonoperative interventions, surgical fusion of the joint can be performed to treat the pain generator (1-7). Minimally invasive SI fusion is intended to stabilize and fuse the SI joint and treat degenerative sacroiliitis. The procedure has increased in popularity for patients who have exhausted less invasive treatment options since the development of a percutaneous approach was first reported in 2004 (1). Possible complications of the procedure include (a) a sacral radiculopathy that can result from compression of the $\mathrm{S} 1$ nerve by the SI joint fusion implant and (b) a lumbar radiculopathy that can result from too ventral a placement of an implant that results in compression of a presacral nerve root. Here we describe 2 cases of minimally invasive endoscopic nerve root decompression surgery that was performed to treat

From: 'Ligamenta Spine Centre, Frankfurt am Main, Germany; ${ }^{2}$ Department of Neurosurgery, Rhode Island Hospital, The Warren Alpert Medical School of Brown University, Providence, RI 
radiculopathy that occurred secondary to minimally invasive placement of SI joint fusion implants.

\section{CASES}

\section{Case 1}

\section{History and Presentation}

A 60-year-old woman underwent a minimally invasive right SI joint fusion for SI pain, adjacent to her instrumented lumbar fusion, that responded well to injections. Post SI joint fusion, the patient complained of right $\mathrm{S1}$ radicular pain and was found to have trace plantar flexion weakness on exam. A computed tomography (CT) scan was performed and indicated that the more cranial SI joint titanium fusion implant had breached the sacral canal; the implant and possibly a cortical bone fragment were likely compressing the $\mathrm{S} 1$ nerve (Fig. 1). The patient underwent an endoscopic procedure which included (a) a laminectomy, (b) drilling down a portion of the compressive titanium SI joint fusion implant, and (c) removal of a cortical bone fragment that was pressing on the $\mathbf{S 1}$ nerve. The patient's radicular symptoms improved immediately, and she remained asymptomatic at the one-year follow-up.

\section{Operative Procedure}

For the endoscopic sacral laminectomy procedure, the patient was positioned in the prone position on a Kambin frame with flexed hips and knees. The procedure was done under general anesthesia. The Joimax iLESSYS $®$ Delta endoscope was used for the procedure. Anteroposterior (AP) and lateral fluoroscopy were used intermittently throughout the case. A one-cm incision was made $2 \mathrm{~cm}$ right of midline (location determined by fluoroscopy) with a scalpel. Under fluoroscopic guidance, a Jamshidi needle and then sequential dilators were used to target the $\mathrm{S} 1$ lamina as a starting point and the final $11.5-\mathrm{mm}$ tubular retractor was inserted. At this point, the Joimax (3M, Saint Pail, MN) rigid laminoscope with a 10 -mm outer diameter and 6-mm working channel was inserted through the tubular retractor (Fig. 1). Under endoscopic view, laminar decompression and reduction of the titanium implant could be achieved using the high-speed endoscopic drill ("Shrill" from Joimax [3M, Saint Pail, MN]) with a $4.5-\mathrm{mm}$ outer diameter head (Fig. 1). A straight grasper was used to remove the cortical bone fragment found immediately adjacent to the titanium implant and compressing the right $\mathrm{S1}$ nerve. In order to avoid a dural tear, meticulous dissection of the interface between the titanium implant and nerve root was performed with a blunt dissector (Fig. 1). Hemostasis was achieved with a radiofrequency probe. Once the bone fragment was removed and the titanium implant was sufficiently reduced in size by drilling, the working channel and scope were removed, pressure was held on the incision for 5 minutes, and the wound was closed with a single suture.

\section{Postoperative Course}

The postoperative course was uneventful, and the patient's radicular pain improved immediately after surgery. One year after her endoscopic procedure, the patient had no clinical symptoms related to the $S 1$ nerve root compression and was symptomatically improved from her SI pain.

\section{Case 2}

\section{History and Presentation}

A 62-year-old man underwent a minimally invasive instrumented fusion from L4 to $\mathrm{S} 1$. After surgery he complained about right-sided iliosacral pain. He underwent a diagnostic block of the SI joint with a positive result. He declined a denervation procedure but agreed to a $\mathrm{SI}$ joint fusion. After his SI joint fusion, he complained of symptoms referable to a right $L 5$ radiculopathy. $A$ CT was performed and showed anterior position of the implant (Fig. 2). The implant was revised. After his SI joint fusion revision procedure, the patient returned 3 weeks later with a persistent L5 radiculopathy. An L5 selective nerve root block completely relieved the pain, but only temporarily. Another CT was performed and showed bone pushing from the lateral to the $L 5$ nerve on the right side (Fig. 2). The patient underwent an endoscopic procedure to remove the bone compressing the $L 5$ nerve. The previous ventrally-directed SI joint fusion implant tract (Fig. 2) was used to access the fragments of bone (Fig. 2). The patient's radicular symptoms improved immediately, and he remained asymptomatic at the one-year follow-up.

\section{Operative Procedure}

The endoscopic surgery was performed using the original SI joint fusion implant tract to gain access to the cortical bone fragments compressing the right L5 nerve (Fig. 2). The patient was positioned in the lateral position under general anesthesia. An 8-mm incision was made along the cranial portion of the former wound and the 


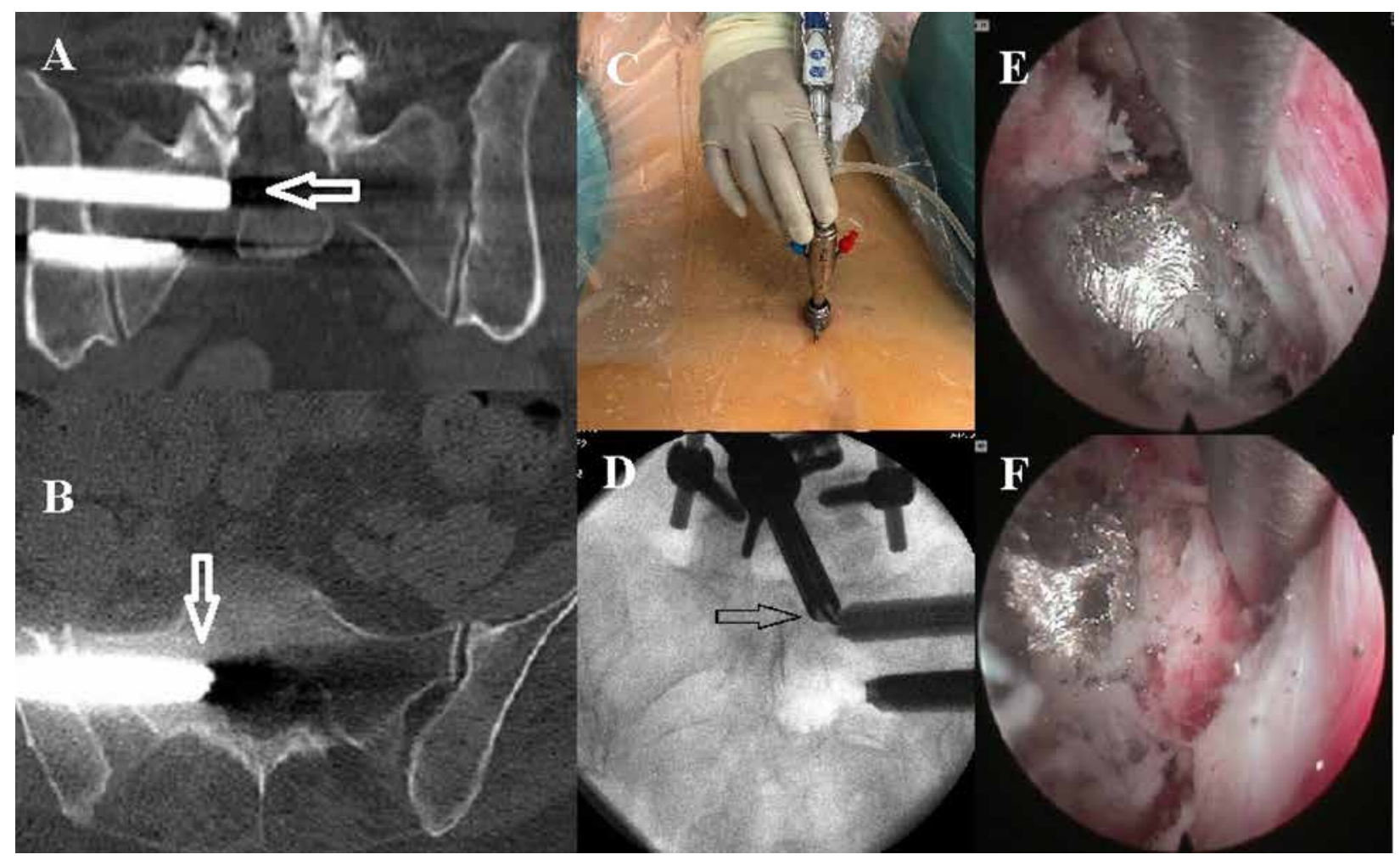

Fig. 1. S1 nerve root compression after a right SI joint fusion procedure. A. Coronal CT reconstruction shows the more cranial $\mathrm{SI}$ joint implant in the sacral foramen where it is compressing the right S1 nerve. B. Axial CT image illustrating the more cranial SI joint implant extending into the sacral canal. C. Photograph of the one-cm diameter working channel endoscope and tubular retractor inserted with fluoroscopic guidance at the lumbosacral junction. D. AP fluoroscopic images depicting the endoscopic dissector placed between the S1 nerve root and the SI joint fusion implant (arrow). E. Endoscopic camera view shows endoscopic dissector retracting the S1 nerve off the SI joint fusion implant. F. Endoscopic camera view shows the endoscopic dissector revealing the compressive fragment of cortical bone revealed after drilling the tip of the implant (the fragment was then removed with an endoscopic grasper).

Abbreviations: AP, anteroposterior; CT, computed tomography; SI, sacroiliac

surgeon was able to feel the previous implant channel with a bullet-shaped $6-\mathrm{mm}$ dilator and then introduce the beveled tubular retractor over the dilator. The access was transiliac and, with continuous endoscopic visualization, the presacral fragments of bone compressing the L5 nerve root could be visualized and removed (Fig. 3). Once the bone fragments were removed, the working channel and scope were removed, pressure was held on the incision for 5 minutes, and the wound was closed with a single suture. Figure 3 demonstrates the endoscopic camera views of: A. a large pre-sacral cortical bone fragment, B. removal of the large cortical bone fragment with an endoscopic grasper, $C$. the decompressed $L 5$ nerve, and D. a zoomed-out view of the bony tract used to access the compressive pathology.

\section{Postoperative Course}

The postoperative course was uneventful, and the patient's radicular pain improved immediately after surgery. One year after his endoscopic procedure, the patient had no clinical symptoms related to the $L 5$ nerve root compression and was symptomatically improved from his SI pain.

\section{DISCUSSION}

Chronic low back pain is a problem frequently encountered in primary care settings and the SI joint has been implicated as a source of chronic low back pain in $15 \%$ to $30 \%$ of patients (2-3). SI joint arthrodesis was originally described in the early 1920 s by Smith-Peterson (4) and performed as an invasive, open approach with minimal to moderate effectiveness (5). Minimally 


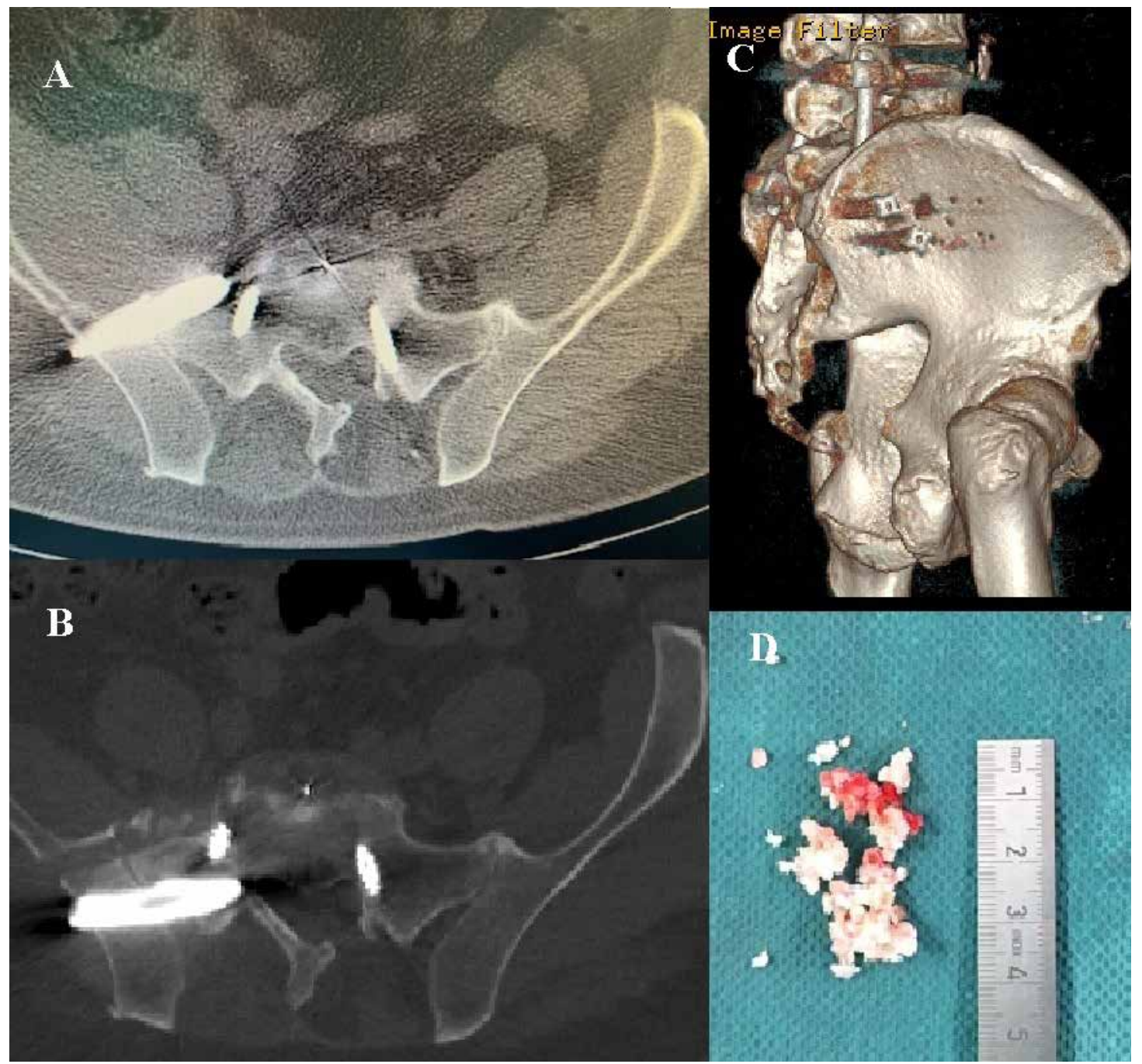

Fig. 2. Ventrally placed SI joint implant. A. Axial CT image demonstrating ventrally placed right-sided SI joint implant (arrow). B. Axial CT image demonstrating tract and cortical bone fragments (arrow) after removal of ventrally placed SI joint fusion implant. C. 3D CT sagittal reconstruction demonstrating the SI joint implants after revision surgery and the tract (arrow) from the previous ventrally placed SI joint fusion implant, which has now been removed. D. Photograph of the cortical bone fragments removed from the endoscopic decompression of bone fragments compressing the right $L 5$ nerve presacrally.

Abbreviations: AP, anteroposterior; CT, computed tomography; SI, sacroiliac

invasive techniques for SI joint fusion have been associated with decreased perioperative morbidity and have increased the popularity of the technique (6-7).

A recent literature review on the safety profile of percutaneous minimally invasive SI joint fusion looking at 14 studies that included 720 complications found a complication rate of $11.1 \%$ (8). The most common complication was wound infection, but $3.05 \%$ of the complications were secondary to placement of the implant, with nerve root impingement being the most common result. The revision rate was $2.56 \%$. The location of the $\mathrm{SI}$ joint places the lower lumbar and sacral nerves at risk during implant placement. The complication is typically corrected by taking the patient back to the operating room to revise the implant. In the 2 cases presented here, cortical bone fragments were 


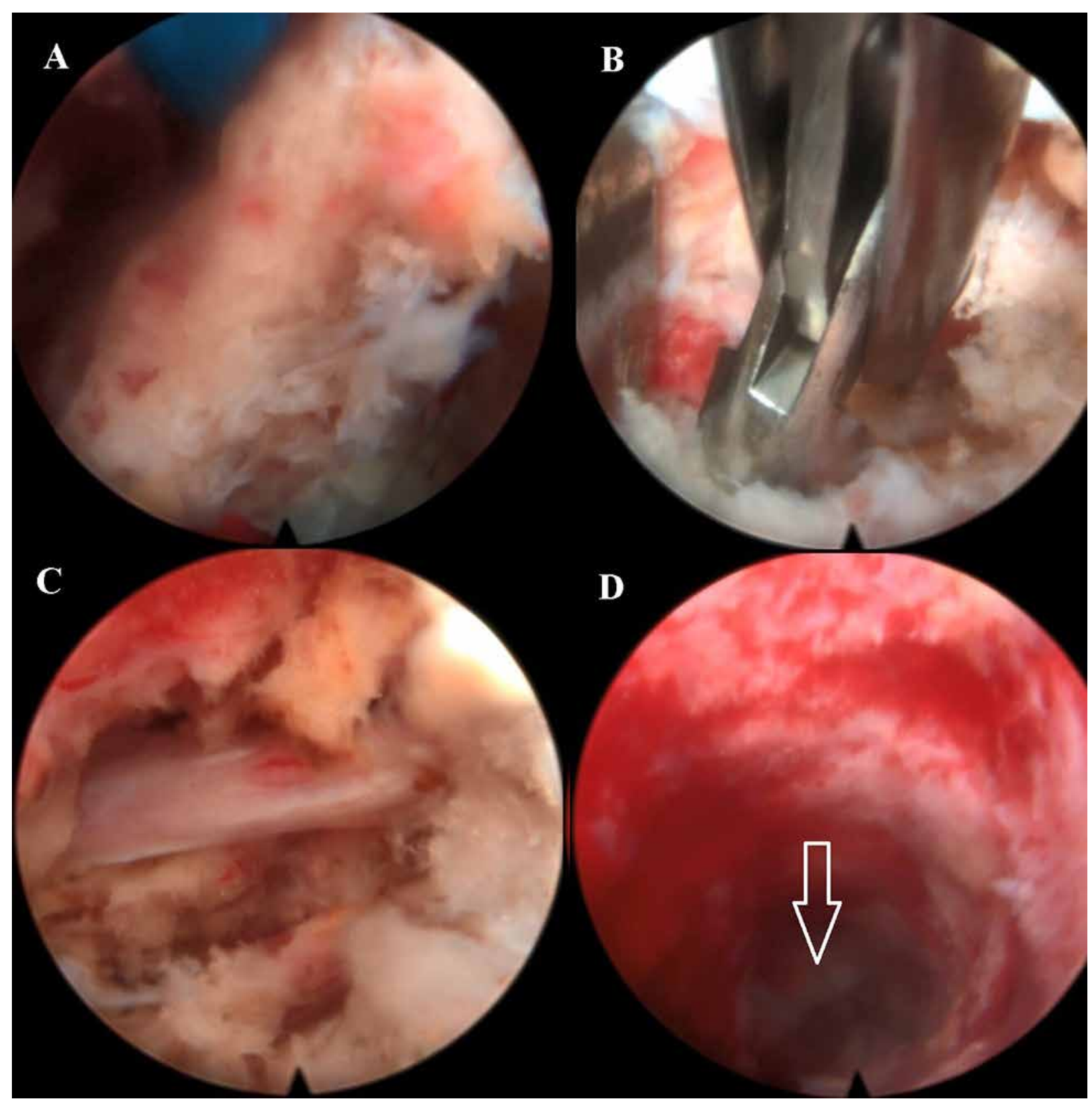

Fig. 3. Endoscopic camera views of decompression of presacral cortical bone fragments compressing the right $L 5$ nerve. A. Cortical bone fragment seen in presacral space. B. Endoscopic grasper removing cortical bone fragment seen in A. C. Decompressed L5 nerve root seen immediately after decompressing cortical bone fragment seen in A. and B. D. Zoomedout view of $\mathrm{C}$ demonstrating the bony channel used to access the presacral space.

significant contributors to nerve compression; even removing and replacing the implant, in the second case presented, did not satisfactorily remedy the patient's radicular symptoms.
In the first case presented here, an endoscopic sacral laminectomy was performed to remove the cortical bone fragment and drill down the portion of the implant that was compressing the nerve. This 
case was published previously as a technical note (9). The 2 cases are presented as a series to illustrate the possible future value of endoscopic spine surgical techniques as a rescue procedure for nerve root compression that can occur as a result of implant positioning in minimally invasive SI joint fusion procedures.

\section{CONCLUSION}

As minimally invasive spine surgical treatments advance and become more prevalent, endoscopic spine surgery "rescue" techniques may also become more prevalent as innovative, minimally invasive treatment options for treating complications associated with minimally invasive spine surgery.

\section{REFERENCES}

1. Giannikas KA, Khan AM, Karski MT, Maxwell HA. Sacroiliac joint fusion for chronic pain: A simple technique avoiding the use of metalwork. Eur Spine J 2004; 13:253-256.

2. Bernard TN Jr, Kirkaldy-Willis WH. Recognizing specific characteristics of nonspecific low back pain. Clin Orthop Relat Res 1987; 217:266-280.

3. Chan KF. Musculoskeletal pain clinic in Singapore-sacroiliac joint somatic dysfunction as cause of buttock pain. Ann Acad Med Singapore 1998; 27:112-115.

4. Smith-Petersen MN. Arthrodesis of the sacroiliac joint: A new method of approach. J Bone Joint Surg 1921; 3:400-405.

5. Spiker WR, Lawrence BD, Raich AL, Skelly AC, Brodke DS. Surgical versus injection treatment for injection - confirmed chronic sacroiliac joint pain. Evid Based Spine Care J 2012; 3:41-53.
6. Smith AG, Capobianco R, Cher D, et al. Open versus minimally invasive sacroiliac joint fusion: A multi-center comparison of perioperative measures and clinical outcomes. Ann Surg Innov Res 2013; $7: 14$.

7. Ledonio CGT, Polly DW Jr, Swiontkowski MF. Minimally invasive versus open sacroiliac joint fusion: Are they similarly safe and effective? Clin Orthop Relat Res 2014; 472:1831-1838.

8. Shamrock AG, Patel A, Alam M, Shamrock KH, Al Maaieh M. The safety profile of percutaneous minimally invasive sacroiliac joint fusion. Global Spine J 2019; 9:874-880.

9. Wagner R, Telfeian AE. An endoscopic surgical technique for treating radiculopathy secondary to $\$ 1$ nerve compression from a pedicle screw: Technical note. J Spine Surg 2018; 4:787-791. 